\title{
Pharmaceutical-grade Oral Films as Substrates for Printed Medicine ${ }^{1}$
}

M. Wimmer-Teubenbacher ${ }^{1}$, C. Planchette ${ }^{2}$, H. Pichler ${ }^{1}$, D. Markl ${ }^{1,3}$, W.K. Hsiao ${ }^{1}$, A. Paudel ${ }^{1,4}$, S. Stegemann $n^{4,5^{*}}$

${ }^{1}$ Research Center Pharmaceutical Engineering GmbH, Graz, Austria

${ }^{2}$ Graz University of Technology, Institute of Fluid Mechanics and Heat Transfer, Graz, Austria

${ }^{3}$ Strathclyde Institute of Pharmacy and Biomedical Sciences, University of Strathclyde, Glasgow, G4 ORE, UK

${ }^{4} \mathrm{Graz}$ University of Technology, Institute of Process and Particle Technology, Graz, Austria

${ }^{5}$ Lonza, Rijksweg 11, 2880 Bornem, Belgium

*Corresponding author: Carole Planchette, E-mail address: carole.planchette@tugraz.at

\section{Abstract}

In contact-less printing, such as piezo-electric drop on demand printing used in the study, the drop formation process is independent of the substrate. This means that having developed a printable formulation, printed pharmaceutical dosage forms can be obtained on any pharmaceutical grade substrate, such as polymer-based films. In this work we evaluated eight different oral films based on their suitability as printing substrates for sodium picosulfate. The different polymer films were compared regarding printed spot morphology, chemical stability and dissolution profile. The morphology of printed sodium picosulfate was investigated with scanning electron microscopy and optical coherence tomography. The spreading of the deposited drops was found to be governed by the contact angle of the ink with the substrate. The form of the sodium picosulfate drops changed on microcrystalline

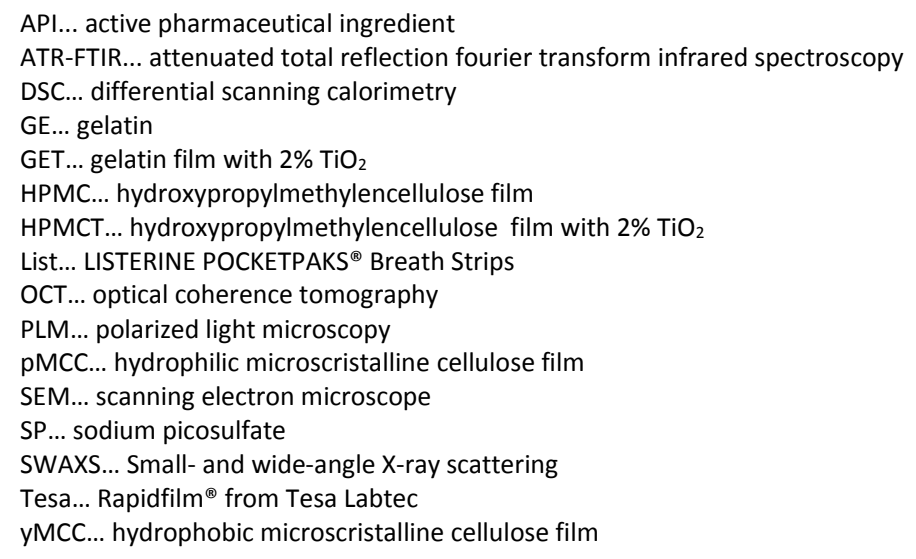


cellulose films at ambient conditions over 8 weeks and stayed unchanged on other tested substrates. Sodium picosulfate remained amorphous on all substrates according to small and wide angle X-ray scattering, differential scanning calorimetry and polarized light microscopy measurements. The absence of chemical interactions between the drug and substrates, as indicated by infrared spectroscopy, makes all tested substrates suitable for printing sodium picosulfate onto them.

Key words: ink jet printing, orodispersible film, sodium picosulfate, dissolution

\section{Introduction}

In the past decade printing has advanced to an enabling technology in many fields of science and engineering (Berthuy et al., 2016; Kastner et al., 2017; Li et al., 2016; Wittbrodt and Pearce, 2017). This trend has also arrived in pharmaceutical manufacturing research since printing of medicines has some specific advantages over other technologies (Boehm et al., 2014). Printing enables a versatile adjustment of the dose by varying the amount of the printed ink which contains the API. Furthermore, it offers the possibility to print a combination of inks and therefore APIs onto a single dosage form to enable personalized drug delivery (Alomari et al., 2015; Planchette et al., 2016). Additionally, the printing process is largely independent from the substrates used when contactless technologies such as ink jet printing systems are used (Daly et al., 2015).

Although inks containing API(s) can be printed onto a wide range of substrates, the substrate attributes such as disintegration time, wettability and mucoadhesion are crucial for the performance of the final dosage form and must be adapted to the intended administration route and desired drug release profile. For printed oral films the gastro- 
intestinal and oromucosal delivery are the primary options (Sandler and Preis, 2016). Films designed for the gastro-intestinal route can either be orodispersible or placed into a capsule targeting the gastro-intestinal tract (Fraser et al., 2013; Pardeike et al., 2011; Preis et al., 2015). Classically used substrates are edible films such as potato starch and rice paper, icing sheet, pure HPMC films, chitosan films or cast films with polymers and plasticizers used in pharmaceutical products (Buanz et al., 2015; Genina et al., 2013; H. Hsu, S. Toth, G. Simpson, L. Taylor, 2013; Janßen et al., 2013; Vakili et al., 2016). Planchette et al. 2016 also printed onto commercially available pharmaceutical orodispersible substrates such as Tesa RapidFilm $^{\circledR}$ (Tesa Labtec GmbH, Langenfeld, Germany) and two MCC films (Cure Pharmaceutical Corp., Oxnard, USA). While it is known that substrate properties like disintegration time, wettability and mucoadhesion influence the patient acceptance, less attention has been given to the interactions of the substrate with the drug. Yet, these interactions are expected to govern the distribution of the printed ink, to possibly cause chemical binding or degradations and to impact the solid form of the drug. Since the solid state form of the drug, crystalline or amorphous, influences the solubility and therefore bioavailability, it is of utmost importance to control it (Lust et al., 2013; Martínez et al., 2014). Classically, this is achieved by employing appropriate excipients inhibiting crystallization (Edueng et al., 2017; Zhang et al., 2014). In printed medicines, it is a crucial point since the manufacturing starts from liquid inks - often containing dissolved APIs. To avoid unwanted API solid states, a strategy consists in employing a substrate that prevents their crystallisation.

The aim of this study was to investigate the influence of printing substrates on the solid form and morphology of ink jet printed sodium picosulfate. Tested dosage forms were produced by printing SP (Sodium Picosulfate) onto 8 substrates typically used for oral 
delivery. The composition of each substrate is detailed in Table 1 of the supplemental information. Beside two commercially available substrates, two microcrystalline cellulose (MCC) substrates differing in excipients other than MCC and two HPMC and two gelatin films both with and without titanium dioxide were studied. Investigated substrate properties were roughness, wettability and chemical composition and the influence thereof on dot size, dot shape, solid state and extractability of printed SP.

\section{Materials and Methods}

\subsection{Films}

Eight different substrates have been investigated in this work. Films fabricated from HPMC, HPMC plus $2 \% \mathrm{TiO}_{2}$ (HPMCT), gelatin (GE) and gelatin plus $2 \% \mathrm{TiO}_{2}(\mathrm{GET})$ were provided by Capsugel (Colmar, France). Hydrophobic and hydrophilic microcrystalline cellulose (pMCC and yMCC, respectively) films were made available by Cure Pharmaceutical Corp. (Oxnard, USA). Tesa RapidFilm ${ }^{\circledast}$ (Tesa) was provided by Tesa Labtec GmbH (Langenfeld, Germany). Listerine PocketPaks Breath Strips (List) (Listerine ${ }^{\circledR}$, Johnson \& Johnson, New Brunswick, New Jersey, USA) were purchased. A table presenting the compositions of the films can be found in supplemental data. All the other chemicals used were of analytical grade. Furthermore, the substrates surface density was measured by weighting samples of $10 \times 30 \mathrm{~mm}^{2}$ and was found to be between 110 and $140 \mu \mathrm{g} / \mathrm{mm}^{2}$ for all substrates.

\subsection{Ink formulation}

Sodium picosulfate (SP) was purchased from Chemos GmbH (Regenstauf, Germany). An aqueous solution of SP with a concentration of $200 \mathrm{mg} / \mathrm{ml}$ was prepared by dissolving the API in purified water (MilliQ, Millipore, Darmstadt, Germany) and a subsequently filtering 
with a $0.45 \mu \mathrm{m}$ Nylon syringe filter (Roth $\mathrm{GmbH}$, Karlsruhe, Germany). This ink formulation has been used for all the experiments described in this work.

\subsection{Printing}

The samples were printed with a piezoelectric printer with a borosilicate glass piezo dispensing capillary with an orifice diameter of $80 \mu \mathrm{m}$ (SciFLEXARRAYER S3, Scienion GmbH, Berlin, Germany). SP was jetted onto the eight substrates at a peak voltage of $83-86 \mathrm{~V}, 300$ $\mathrm{Hz}$ and a pulse of $50 \mathrm{~ms}$ forming drops with an average drop volume of $430 \mathrm{pl}$ for a standard deviation of 1.5\% (Planchette et al., 2016) except otherwise specified. Different patterns were printed for different testing methods. The drops were deposited in squares of $5 \times 5 \mathrm{~mm}^{2}$ with a varying number of isolated spots per square. For optical coherence tomography, scanning electron microscopy and polarized light microscopy measurements: 100 (10X10) spots, separated by $500 \mu \mathrm{m}$ and consisting of 20 drops per spot were printed corresponding to a total ink volume of $860 \mathrm{~nL}$ and a total dose of $172 \mu \mathrm{g}$ of SP for approximately $3500 \mu \mathrm{g}$ of substrate. For HPLC and SWAXS measurements, printed samples consisted of 625 (25X25) spots, separated by $100 \mu \mathrm{m}$ with 15 drops per spot providing a printed ink volume of $4.03 \mu \mathrm{l}$ providing $806 \mu \mathrm{g}$ of SP for approximately $3500 \mu \mathrm{g}$ of substrate. DSC samples were printed with $43 \times 43$ spots with 16 drops per spot on $5 \times 5 \mathrm{~mm}^{2}$ of substrate to reach an amount of 2.5 $\mathrm{mg}$ of SP for about $3.5 \mathrm{mg}$ of substrate. For the dissolution tests drops of approximately 360 pL were printed. 6000 spots $(200 \times 30)$ consisting of 21 drops per spot enabled to deposit 45 $\mu \mathrm{L}$ of ink corresponding to $9 \mathrm{mg}$ of SP on $2 \times 3 \mathrm{~cm}^{2}$ corresponding to approximately $85 \mathrm{mg}$ of substrate.

\subsection{Scanning electron microscopy}


SEM images were taken with a JEOL JSM 5600 (JEOL Ltd., Tokyo, Japan) with an adjusted acceleration of $7 \mathrm{kV}$. Before analysis the samples were prepared by aluminum sputtering in vacuum. The eight substrates were imaged before printing, just after printing and after printing with a storage period of 8 weeks at room temperature and humidity.

\subsection{Optical coherence tomography}

A spectral-domain (SD-)OCT system was used to create 3D images combining 512 depthresolved cross-section images (OCT Pharma 3D, Phyllon GmbH, Graz, Austria). A SLD Broadlighter was used as light source. The following settings were used during acquisition: central wavelength of $830 \mathrm{~nm}$, full width at half maximum (FWHM) bandwidth of $75 \mathrm{~nm}$, lateral resolution of $\sim 10 \mu \mathrm{m}$, axial resolution of $\sim 4 \mu \mathrm{m}$, and single depth-scan acquisition rate of $30 \mathrm{kHz}$ (Markl et al., 2014). The dosage forms obtained on the eight investigated substrates were imaged just after drying of the printed ink and after having been stored for 8 weeks at room conditions.

\subsection{Polarized light microscopy}

Microscopy images were obtained with 4x magnification (SENTERRA II, Bruker Optic GmbH, Ettlingen, Germany). A U-PO3 polarizer slider and U-AN 360-3 rotatable analyzer slider (Olympus, Tokyo, Japan) were used to create polarized light images. A THMS 600 hot stage was used from Linkam (Linkam Scientific Instruments, Tadworth, UK). Hot stage microscopy was conducted in two stages: $100 \mathrm{~K} / \mathrm{min}$ form 20 to $100^{\circ} \mathrm{C}, 10 \mathrm{~K} / \mathrm{min}$ from 100 to $300^{\circ} \mathrm{C}$ with stable temperatures at $140,180,250$ and $300^{\circ} \mathrm{C}$ to take a picture for $1 \mathrm{~min}$. The dosage forms obtained for each of the 8 substrates were imaged just after drying of the printed ink and after having been stored for 14 days at $40^{\circ} \mathrm{C}$. 


\subsection{Contact angle measurement}

The sessile drop method (EasyDrop, Krüss $\mathrm{GmbH}$, Hamburg, Germany) was used to measure the contact angle (liquid/solid interface) made by a $1 \mu \mathrm{SP}$ ink drop (diameter $1.24 \mathrm{~mm}$ ) placed on the film surface. All measurements were made tenfold, at room temperature, within 30 seconds after drop deposition and the average values were calculated.

\subsection{Differential scanning calorimetry}

Differential scanning calorimetry (DSC) measurements were performed using a DSC-204 F1 Phoenix (Erich Netzsch GmbH \& Co. Holding KG, Selb, Germany) on freshly printed samples with a mass of about $2.5 \mathrm{mg}$ of sodium picosulfate on a surface area of $5 \mathrm{~mm}^{2}$ creating a total sample weight of about $5.0 \mathrm{mg}$, at a heating rate of $10 \mathrm{~K} / \mathrm{min}$, and at a temperature range from room temperature to $310^{\circ} \mathrm{C}$.

\subsection{Small- and wide-angle X-ray scattering}

Small- and wide-angle X-ray scattering (SWAXS) measurements were conducted using a SWAXS- Hecus S3-Micro system (Hecus X-Ray Systems GmbH, Graz, Austria) operating at 50 $\mathrm{kV}$ and $1 \mathrm{~mA}$. Samples consisting of approximately $800 \mu \mathrm{g}$ of SP on $3500 \mu \mathrm{g}$ of substrate were measured in transmission mode with wolfram $27050 \mu \mathrm{m}$ filter position for $30 \mathrm{~min}$. The scattering angles were from $0-9^{\circ}$ and $18-27^{\circ}$ for SAXS and WAXS, respectively.

Temperature resolved SAXS measurements were performed using a point-focusing camera system with a DSC (MicroCaliX, Bruker AXS, Karlsruhe, Germany) powered by a Cu microsource with a wavelength of $1.542 \AA$ at room temperature $\left(20^{\circ} \mathrm{C}\right)$, with a real space resolution range from 10 to $100 \AA$. The sample was heated with a heating rate of $5 \mathrm{~K} / \mathrm{min}$ and from room temperature to $180^{\circ} \mathrm{C}$. The measurements were conducted from 80 to 
$180^{\circ} \mathrm{C}$ and each measurement was taken for a range of $20 \mathrm{~K}$ equivalent to $4 \mathrm{~min}$ measurement time.

\subsection{Content analysis of printed API}

SP was extracted from the dosage forms consisting of printed and dried ink on the substrates. The substrates were cut to $2 \times 3 \mathrm{~cm}^{2}$ strips and $0.05 \mathrm{ml}$ of the SP ink with a concentration of $200 \mathrm{mg} / \mathrm{ml}$ were deposited onto the substrates resulting in approximately 9 mg of SP on $85 \mathrm{mg}$ of substrate. Dosage forms made with each of the eight substrates were investigated just after production and after storage for 14 days at $40^{\circ} \mathrm{C}$. The extraction was carried out in $10 \mathrm{ml}$ of aqueous $2 \%$ acetonitrile solution at room temperature (RT) and 200 $\mathrm{rpm}$ for $2 \mathrm{~h}$. Gelatin samples were extracted at $42^{\circ} \mathrm{C}$. The extracted samples were centrifuged instead of filtration and $20 \mu \mathrm{l}$ was injected into the HPLC. SP content of the printed samples was determined by using HPLC with UV-detection. Flow rate was set to 0.6 $\mathrm{ml} / \mathrm{min}$, injection temperature at $20^{\circ} \mathrm{C}$, column temperature at $30^{\circ} \mathrm{C}$, and detection wavelength was $264 \mathrm{~nm}$. A 98:2 mixture of $10 \mathrm{mM}$ ammoniumformat buffer $\mathrm{pH} 3$ and acetonitrile was used as mobile phase for $8 \mathrm{~min}$ with a pulse of 5:95 mixture for $10 \mathrm{~s}$ and 98:2 up to 14 min. A Waters Alliance 2695 (MassLynx) (Waters Cooperation, Milford, MA, USA) equipped with a 100 RP-18 endcapped column (Merck, Supersher 100 RP-18 endcapped column, LiChroCART 125-4 + Guard Column 5 $\mu \mathrm{m}$ ) was used. Linearity was proven for concentrations between 60 and $100 \mu \mathrm{g} / \mathrm{ml}$ with $\mathrm{R}^{2}=0.9999$. All measurements were performed in triplicate.

\subsection{In vitro evaluation of drug release}

Drug release tests were performed in $250 \mathrm{ml}$ phosphate buffer $\mathrm{pH} 6.0$ (buffer formulation according to Ph.Eur. 6.2 dissolution test for medicated chewing gum EMA, 2013), $37^{\circ} \mathrm{C}$ and 
50 rpm (Garsuch and Breitkreutz, 2009). A USP type 2 apparatus (DT820LH, ERWEKA GmbH, Heusenstamm, Germany) was used to conduct the tests. The samples, SP printed onto the eight substrates, stored at ambient conditions for a maximum of three days after printing, were placed into the dissolution medium and $1 \mathrm{ml}$ was removed at predetermined time intervals. The buffer was replenished to maintain the original dissolution volume of $250 \mathrm{ml}$. The withdrawn samples were centrifuged at $15.000 \mathrm{rpm}, 20^{\circ} \mathrm{C}$ for $10 \mathrm{~min}$, to remove residual particles and the SP concentration was determined using the HPLC method described in 2.10. All dissolutions were carried out in triplicate.

\subsection{ATR-FTIR}

A VERTEX 70 FTIR (Bruker Optic GmbH, Ettlingen, Germany) with a MIRacle ${ }^{\text {TM }}$ ATR (PIKE Technologies, Madison, WI, USA) was used to measure infrared spectra of the films, API powder, and printed samples right after production and after storage for $14^{\circ} \mathrm{C}$ at $40^{\circ} \mathrm{C}$. For MCC films, further measurements were performed using physical mixtures of the films and API powder. In order to create the physical mixtures, films and SP (1:1 weight ratio) were pestled in a porcelain mortar. Per measurements 64 spectra were recorded at a spectral range of 600 to $4000 \mathrm{~cm}^{-1}$. All measurements were performed in triplicate.

\section{Results}

\subsection{Morphological investigation using SEM}

SEM pictures of all eight substrates were taken before printing, after SP spots were freshly printed (Figure 1) and after storage at ambient conditions $\left(19-25^{\circ} \mathrm{C}, 40 \% \mathrm{RH}+/-20 \% \mathrm{RH}\right)$ for 8 weeks (Figure 3). The substrate surfaces can be classified as rather smooth or rather rough. List, the gelatin and HPMC films are smoother than the Tesa and MCC films appeared 
to have rougher surfaces. yMCC was found to be porous (Planchette et al., 2016) which was even more apparent once SP was printed onto the substrate (Figure 1).

Figure 1: SEM pictures of ; $1^{\text {st }}$ column blank substrates (top to bottom): Tesa, HPMC, GE, yMCC; $2^{\text {nd }}$ column after printing SP onto the substrates (top to bottom): Tesa, HPMC, GE, yMCC;; $3^{\text {rd }}$ column blank substrates (top to bottom): List, HPMCT, GET, pMCC; $4^{\text {th }}$ column after printing SP onto the substrates (top to bottom): List, HPMCT, GET, pMCC.

The roughness of the surface had an influence on the shape of the ink drops. On the smooth films the drops formed spots with smooth edges, as on HPMC or gelatin films. In contrast and as expected, irregular spot shapes were observed on rough substrates due to the pinning of the contact line at the physical and chemical irregularities (Quéré, 2008). Spots edges were found to follow the structure of the film as it can be seen for the MCC substrates (Figure 1). SP spots formed irregularly shaped edges on Tesa as well. The irregularities of the SP spot edges were smaller than on the MCC films which can be explained by the smaller magnitude of the surface roughness on the Tesa film. Further to the edges of the spots, the different films influenced the size of the ink spots as well. Figure 2a shows $S_{m}$ the average spot areas of SP ink printed onto the different substrates as a function of their contact angle. In general, SP ink formed smaller sized spots with increasing contact angles and therefore, decreasing wettability of the substrates. Knowing $V$, the volume of each spot and assuming the substrates are non-porous and non-dissolving solid surfaces, we derive by applying the volume conservation principle to a spherical cap having a contact angle $\theta$, the theoretical diameter of the printed spots, $d_{\text {theo }}$. The detailed derivation is given in the supplemental information, for completeness. We obtain for both hydrophilic $\left(\theta<90^{\circ}\right)$ and hydrophobic $\left(\theta>90^{\circ}\right)$ substrates: 


$$
d_{\text {theo }}=(6 V / \pi)^{1 / 3} 2 \sin \theta(4+\cos \theta(\cos (2 \theta)-5))^{-1 / 3}
$$

Measured spot diameters $d_{m}=2\left(S_{m} / p\right)^{1 / 2}$ are plotted against $d_{\text {theo }}$ the predicted values obtained with eq. (1) on Fig. 2b. The results are in good agreement except for the List and the HPMCT films where SP drops were bigger than their contact angles would suggest. The discrepancies could originate the local dissolution of the films. If the dissolution kinetics is fast with respect to the typical evaporation timescale of one spot, the effective volume of liquid material $\mathrm{V}_{\text {eff }}$ will be larger than the one of the deposited ink $\mathrm{V}$. The size of the spot will consequently be increased by a factor of $\left(\mathrm{V}_{\text {eff }} / \mathrm{V}\right)^{1 / 3}$. Additionally, some components of the films (surfactants/surface active particles) could be solubilized/ dispersed in the freshly printed spot and promote its spreading. As the spot volume being approximately 100 times smaller than the volume of the drop used for contact angle measurement, this effect could have been underestimated. Further investigations would be needed to test these two hypotheses. Finally, possible spot coalescence was attributed to contact between adjacent spots. The spot spacing set in the printing pattern remaining unchanged, this phenomenon is expected to become more frequent for increasing spot spreading and thus for increasing substrate wettability. Experimental observations confirm this interpretation. Indeed, on GE, HPMCT, HPMC and List drops coalesced with neighboring drops and formed double, triple and even quadruple drop spots. The phenomenon is especially pronounced for List for which the theoretical spot diameter after spreading $d_{\text {theo }}$ reaches $500 \mu \mathrm{m}$, the set spot spacing.

Figure 2: a) contact angles of SP on the eight substrates plotted against surface areas of printed SP dots, Grubb's outlier test has been performed on the results for the contact angles, error bars are standard deviations ( $n=10), b)$ experimental spot diameters as a function of theoretical ones, black circles: rough surfaces, grey diamonds: smooth surfaces, the dashed line indicates $500 \mu \mathrm{m}$ the distance separating two spots. 
Figure 3: SEM images of SP printed on (a) HPMC, (b) HPMCT, (c) TESA, (d) GE, (e) GET, (f) LIST after storage at room conditions for a period of eight weeks.

Samples printed the same conditions as shown in Figure 1 were investigated with SEM after storage at ambient conditions for 8 weeks (Figure 3). Some of the drops have a rougher surface after storage than before (see Figure 1). Further investigations with polarized light microscopy, DSC and SWAXS were conducted to determine whether these changes in roughness can be attributed to a change in the solid-state of the API (crystalline or amorphous). The most significant changes could be observed for the samples with SP on MCC films. SP dots were no longer visible on these films after storage at ambient conditions for 8 weeks (images not shown). The disappearance of SP dots was further investigated by content analysis with HPLC and ATR-FTIR measurements.

\subsection{Interfacial tomographic analysis by OCT}

Figure 4 depicts OCT images of SP printed on different substrates. The cross-sectional OCT images were acquired at the center of several adjacent spots. The substrate is visible on the lower half of the images whose width corresponds to $3.12 \mathrm{~mm}$, arrows indicate the location of spots. The dots were visible in all OCT images except for yMCC film, the most porous substrate. Similar to the SEM images of List, the spreading of the SP can be observed in the OCT image. The higher contrast of the substrates in

Figure $4 \mathrm{~d}$ and $\mathrm{f}$ (substrate with $\mathrm{TiO} 2$ ) compared to $\mathrm{c}$ and e (substrates without $\mathrm{TiO} 2$ ) is the result of scattering losses induced by $\mathrm{TiO}_{2}$, which are known as highly scattering particles (Lin et al., 2017). 
Figure 4: OCT images of SP freshly printed on (a) HPMC, (b) HPMCT, (c) TESA, (d) GE, (e) GET, (f) LIST, (g) yMCC, (h) pMCC. Optical dimensions (in air) of each image (1024 × 1024 pixels): $3.12 \times 3.12 \mathrm{~mm}^{2}$, images were cropped in y direction without distorting the scale.

Figure 5: OCT images acquired after storing the printed samples for 8 weeks. (a) Tesa, (b) List, (c) HPMC, (d) HPMCT, (e) GE, (f) GET, (g) yMCC, (h) pMCC. Optical dimensions (in air) of each image (1024 × 1024 pixels): $3.12 \times 3.12 \mathrm{~mm}^{2}$, images were cropped in y direction without distorting the scale.

The main difference between fresh samples and samples after storage can be observed for SP printed on pMCC (Figure $5 \mathrm{~h}$ ), where the dots disappeared. This mass transport might be driven by diffusion caused by environmental humidity. However, the dots printed on the other films are broader and thus the SP spread on the substrate.

\subsection{Morphology and amorphous state with PLM}

Figure 6: Microscope images with 4x magnification of SP powder and different printed substrates. SP printed on (a) on Tesa immediately after printing, (b) on Tesa after 14 days at $40^{\circ} \mathrm{C}$, (c) on HPMC immediately after printing, (d) on HPMC after 14 days at $40^{\circ} \mathrm{C}$, (e) on pMCC immediately after printing, (f) on pMCC after 14 days at $40^{\circ} \mathrm{C}$, (g) GET immediately after printing, (h) on GET after 14 days at $40^{\circ} \mathrm{C}$; (i) SP powder, and (j) SP powder under polarized light. Color available online.

Images taken with a light microscope support the observations from the SEM and OCT measurements (

Figure 6 for Tesa, HPMC, pMMC and GET; supplemental information for the other substrates). SP spots tended to coalesce on HPMC, HPMCT, and List, formed smooth 
surfaces on GE and GET and ridges and folds on Tesa, HPMC, and HPMCT. SP did not show any morphological changes after storage at $40^{\circ} \mathrm{C}$ for 14 days except on both MCC films. After 14 days of storage SP dots showed a melted appearance on yMCC and pMCC. In order to detect crystalline areas, we used polarized light (Adler et al., 2015). SP powder and dried aqueous ink on glass slides displayed clearly visible crystalline areas under polarized light (Figure $8 \mathrm{~d}$ ). Interestingly, SP printed on any of the 8 films did not show crystalline areas under polarized light, neither when freshly printed nor after storage at $40^{\circ} \mathrm{C}$ for 14 days (images not shown). Polarized light images could not evidence crystallization of SP on any of the 8 substrates. To confirm the absence of crystallization, further tests were conducted with DSC, hot stage microscopy and SWAXS enabling a deeper probing of the materials.

\subsection{Thermal investigations using DSC and hot stage microscopy}

DSC measurements were conducted to investigate the solid state of printed SP on the substrates. The results which consist of DSC curves of SP powder, dried SP from aqueous ink and SP ink printed onto the substrates, are presented in Figure 7 for the case of gelatin. The DSC curves of powder and dried SP showed decomposition at 302 and $297^{\circ} \mathrm{C}$, respectively. SP powder showed an additional peak with an onset at $146.4^{\circ} \mathrm{C}$ and a maximum at $172^{\circ} \mathrm{C}$ which did not occur in SP from the dried ink. In literature only a decomposition point at 272$275^{\circ} \mathrm{C}$ was reported for SP (H. Hager, W. Blaschek, S. Ebel and Hackenthal, 2003). In SP originating from dried ink a much flatter peak appeared with an onset at $42^{\circ} \mathrm{C}$. When printed onto gelatin SP showed the same flat peak as dried SP ink with an onset at $42^{\circ} \mathrm{C}$. The difference between the curve of GE alone and the one for printed SP on GE demonstrates that the printed amount of SP was sufficient to be detected. To further investigate the presence of the peaks observed with DSC between $42^{\circ} \mathrm{C}$ and $172^{\circ} \mathrm{C}$, SP powder, dried SP from aqueous ink and SP ink printed onto GE were analyzed with hot stage microscopy up to 
$300^{\circ} \mathrm{C}$. Hot stage microscopy was performed with normal and polarized light in duplicate.

The appearance of SP powder did not change during the heating between room temperature and $180^{\circ} \mathrm{C}$ excluding that melting took place from $146.4^{\circ} \mathrm{C}$ on (Figure 8 a and b). Indeed, SP powder remained the same till it began to melt and disintegrate between 260 and $300^{\circ} \mathrm{C}$. The DSC peak with an onset at $146.4^{\circ} \mathrm{C}$ could therefore be caused by the apparition of a mesomorphic structure of the SP. This hypothesis seems in agreement with X-ray measurements, see Figure 11. For SP originating dried ink, melting could be observed under hot stage microscopy between 20 and $140^{\circ} \mathrm{C}$ as displayed in Figure $8 \mathrm{~d}$ and e. Thus the DSC peak observed around $42^{\circ} \mathrm{C}$ could be attributed to melting. In the same temperature range, between 40 and $140^{\circ} \mathrm{C}$, a morphological change could be observed for SP printed on gelatin (Figure $8 \mathrm{~g}$ and $\mathrm{h}$ ) in agreement with the DSC measurements. Furthermore, for SP printed on gelatin, no disintegration was observed below $300^{\circ} \mathrm{C}$.

Figure 7: DSC curves of SP powder, dried aqueous SP ink, freshly printed SP on gelatin and gelatin.

Figure 8: Hot stage microscopy images of SP with and without polarized light. (a) SP powder under polarized light at $20^{\circ} \mathrm{C}$, (b) at $180^{\circ} \mathrm{C}$ and (c) at $300^{\circ} \mathrm{C}$; (d) aqueous SP ink dried on glass under polarized light at $20^{\circ} \mathrm{C}$, (e) at $140^{\circ} \mathrm{C}$ and (f) at $300^{\circ} \mathrm{C}$; (g) SP printed onto gelatin under normal light at $20^{\circ} \mathrm{C}$, (h) $140^{\circ} \mathrm{C}$ and (i) $300^{\circ} \mathrm{C}$; the scale bar applies to all images, color available online.

\subsection{Small- and wide-angle X-ray scattering}

SWAXS measurements were conducted to investigate crystal structures of SP powder, especially to explain the peak observed with DSC around $170^{\circ} \mathrm{C}$, and of PS printed onto the 8 investigated substrates. SP powder showed 12 crystallization peaks within the WAXS curve (Figure 9) and 2 within the SAXS curve (Figure 10). Blank GE film did not show any 
crystallization peaks in WAXS or SAXS curves nor did any of the other 7 blank films except peaks at $\sim 25$ deg corresponding to $\mathrm{TiO}_{2}$ (curves shown in supplemental data). The diffractograms of SP on GE film - both when freshly printed and after storage at $40^{\circ} \mathrm{C}$ for 14 days did not evidence any crystallization peak. This result should be carefully interpreted since it could also be due to the amount of printed SP, possibly to close to the detection limit. These results were also observed on the 7 other films (supplemental data). SWAXS curves confirmed, assuming the limit of SP detection was reached, that no crystallization of the printed SP occurred for any of the films even after storage at $40^{\circ} \mathrm{C}$ for 14 days.

Figure 9: WAXS curves of SP powder, SP printed on GE stored at $40^{\circ} \mathrm{C}$ for 2 weeks, SP printed on GE after printing and blank GE film; logarithmic scale based on 10. Color available online.

Figure 10: SAXS curves of SP powder, SP printed on GE stored at $40^{\circ} \mathrm{C}$ for 2 weeks, SP printed on GE after printing and blank GE film. Color available online.

In order to investigate the behavior of SP powder between 140 and $180{ }^{\circ} \mathrm{C}$ and to explain the peak observed with DSC (Figure 7), additional SAXS measurements on heated powder were conducted. Between 100 and $140{ }^{\circ} \mathrm{C}$ the curves remained similar, whereas between 140 and $180{ }^{\circ} \mathrm{C}$ the peak between number 140 and 145 was elevated by 20 and 30 counts (Figure 11, circled peak). available online. 


\subsection{Content analysis of printed samples}

Figure 12: SP contents of printed samples after storage at $40^{\circ} \mathrm{C}$ for 0 and 14 days, error bars are standard deviation ( $n=3$ ).

SP content assays were performed right after printing SP on the substrates and after storage at $40^{\circ} \mathrm{C}$ for 14 days. The results of the content assays are displayed in Figure 12 . The theoretical contents, $100 \%$ in Figure 12, were calculated using the drop volume determined by the Scienion drop watcher, the amount of printed drops and the SP concentration of the ink. Content assays revealed that the accuracy of printing aqueous SP ink is well within a standard deviation of $\pm 3 \%$ freshly after printing. SP contents decreased slightly on all 8 substrates after storage at $40^{\circ} \mathrm{C}$ for 14 days. The highest decrease in SP content was observed when printed on pMCC where it dropped from $6.9 \%$ followed by List $(5.3 \%)$ and Tesa (4.9\%). On the other substrates the content decrease was less than $5 \%$. After storage at $40^{\circ} \mathrm{C}$ no peaks of degradation products were found in the HPLC chromatograms. ATR-FTIR measurements were conducted for further investigation of possible degradation of SP printed onto the substrates or chemical interactions between API and the films.

\subsection{Chemical interactions investigated with ATR-FTIR}

Figure 13: ATR-FTIR curves of SP powder, MCC films and physical mixtures (1:1) of SP powder and MCC films. (a) SP and yMCC, (b) SP and pMCC. Color available online.

ATR-FTIR spectra were taken to identify chemical interactions between SP and the substrates. The ATR-FTIR spectra were made of SP powder, SP printed onto different 
substrates freshly printed and after storage, and blank substrates. SP displayed the same peaks when printed onto all eight substrates freshly after printing and after storage at $40^{\circ} \mathrm{C}$ for 14 days (spectra are shown in the supplemental data). These findings indicate that there are no significant chemical interactions between SP and the films. Due to the morphological changes observed with PLM, OCT and SEM for PS printed on the MCC films, further investigations have been performed for these films. Therefore, physical mixtures, $1: 1$ of SP and the MCC films were prepared and investigated with ATR-FTIR.

Figure 13 displays the spectra of SP printed onto the MCC films; the band assignments are listed in Table 1 according to (George Socrates, 2004; Romani et al., 2016). As shown in Figure 13, the SP spectra were similar in the physical mixture with both MCC films and pure SP. After 14 days of storage at $40^{\circ} \mathrm{C}$ no chemical changes were detected in printed SP. However, shifts could be observed in the regions of $1279-1259,165-1051$ and, $873-860 \mathrm{~cm}^{-1}$ which have all been assigned to the bonds neighboring pyridines nitrogen.

Table 1: band assignments of ATR-FTIR measurements of SP powder, SP printed onto different substrates according to (George Socrates, 2004; Romani et al., 2016), bands marked with * exhibited changes after storage at $40^{\circ} \mathrm{C}$ for 14 days.

\begin{tabular}{|c|c|c|c|}
\hline band $\left[\mathrm{cm}^{-1}\right]$ & assignment & band $\left[\mathrm{cm}^{-1}\right]$ & assignment \\
\hline $1610-1587$ & $\begin{array}{l}\text { C-C stretching, pyridine and } \\
\text { benzene }\end{array}$ & $1203-1200$ & stretching in $\mathrm{SO}_{3}$-groups \\
\hline 1500 & $\begin{array}{l}\mathrm{C}-\mathrm{H} \text { deformation in plane and } \\
\mathrm{C}-\mathrm{C} \text { stretching, benzene }\end{array}$ & $1088-1086$ & C-O stretching \\
\hline $1467-1433$ & $\begin{array}{l}\mathrm{C}-\mathrm{H} \text { deformation in plane, } \\
\text { pyridine and benzene }\end{array}$ & $1065-1051$ & C-C stretching in pyridine* \\
\hline $1279-1259$ & $\mathrm{~N}-\mathrm{H}$ stretching, pyridine* & $1018-1016$ & ring stretching \\
\hline
\end{tabular}




\begin{tabular}{|l|l|l|}
$1237-1234 \quad$ C-N stretching, pyridine & $\begin{array}{l}873-860 \\
\text { C-C deformation linking the } \\
\text { rings* }\end{array}$ \\
\hline
\end{tabular}

\subsection{In vitro evaluation of drug release}

Figure 14: Comparison of drug release of printed SP from the different substrates; release from (a) GE and GET films, (b) HPMC and HPMCT films, (c) yMCC and pMCC films and (d) Tesa and List films; error bars are standard deviation (n=3).

Printed SP remained on the surface of the different films and was visible to the unattended eye. Despite SP remaining on the surface, the printed samples displayed differences in drug release (

Figure 14). HPMC and GE films resulted in slower release when compared to the MCC films, Tesa and List. HPMC and GE substrates formed a clump at the bottom of the dissolution vessel which was not completely dissolved over the course of $120 \mathrm{~min}$. The drug release from HPMC and HPMCT reached a maximum of 89 and 95\% within 30 min, GE and GET 89 and $93 \%$ within $60 \mathrm{~min}$, respectively. The two commercially available, orodispersible films Tesa and List disintegrated completely within $30 \mathrm{~s}$ and $5 \mathrm{~min}$, respectively. The release of SP reached a plateau of $\sim 98 \%$ when released from Tesa and List within 15 and $30 \mathrm{~min}$, respectively. pMCC films did not disintegrate during the dissolution test but appeared to swell and the shape of individual films remained intact. In contrast, yMCC disintegrated into smaller pieces and formed an opaque dispersion within $5 \mathrm{~min}$. Drug release from pMCC and yMCC reached a maximum of $\sim 97 \%$ within 5 and 15 min, respectively.

\section{Discussion}


In contact-less printing, such as piezo-electric drop on demand printing used in the study, the drop formation process is independent of the substrate. This means that having developed a printable formulation, printed pharmaceutical dosage forms can be obtained on any pharmaceutical grade substrate, such as polymer-based films. In this work we evaluated different substrates based on their suitability as printing substrates for SP. The different polymer films were compared regarding morphology, chemical stability and dissolution. The different substrates tested in this study provide a wide range of contact angles and thus wettability. Contact angles were found to inversely correlate with drop areas of freshly printed samples. Theoretical spot diameters predicted from volume conservation and contact angle agreed very well with the observed values except for List and HPMCT. The discrepancies observed for List and HPMCT can originate the local dissolution of the substrate or the release of surface active agents into the freshly printed spot which modify its spreading. The coalescence of SP ink printed onto the substrates was clearly observed for substrates onto which the theoretical spot diameter was close to or larger than $500 \mu \mathrm{m}$, the set value of spot spacing. The frequency of spot coalescence increased with an increasing $d_{\text {theo. }}$ Numerous coalescence events as observed onto List could also explain the difference between predicted and observed spreading. Thus, except for List and HPMCT, the equations $(1,2)$ can accurately be used to set the minimum spot spacing to avoid spot coalescence for arbitrary spot volumes.

The morphology of the substrate surfaces had an impact on the shapes of the formed dots. Smooth substrates facilitated the formation of circular dots, or dumbbell-shaped when coalesced, with regular edges, while rougher substrates promoted the formation of irregular shaped dots due to the pinning of the contact line. The porosity of yMCC (Planchette et al., 2016) caused the dots of dried ink to vanish from the substrate surface over a course of 
eight weeks at ambient conditions. Genina et al. (2013) argues that porous substrates are better penetrated by the API containing inks and therefore cause entrapment of the API. However, further studies on the correlation between entrapment and porosity are necessary. The hypothesis of SP migrating into the $\mathrm{YMCC}$ matrix is supported by the fact that SP could be recovered from all substrates and no degradation products were found during HPLC measurements. The loss of extracted SP from yMCC could therefore be caused by its diffusion within the film which poorly dissolved during extraction.

SP formed dome shaped dots on the surface of pMCC when freshly printed (Figure 1, Figure 4,

Figure 6) and changed the form over storage (Figure 5, Figure 6). This indicates that as for YMCC, SP migrates into the film during storage. ATR-FTIR measurements did not reveal any chemical reactions between SP and pMCC and no degradation products were detected with HPLC. However, shifts were observed for the bands assigned to $\mathrm{N}-\mathrm{C}$ and $\mathrm{C}-\mathrm{C}$ stretching in the pyridine ring. The shifts can be explained by the drier state of the samples after storage and the resulting loss of hydrogen bonds between the nitrogen in the pyridine ring and water which alters the characteristics of neighboring bonds (Berezin et al., 2004).

This suggests that as for yMCC, the loss of extracted SP could originate its diffusion within the film which does not dissolve during extraction. PMCC being more hydrophobic than yMCC, it was expected and experimentally observed that the pMMC substrate dissolves less efficiently than the film made of yMMC. As a result, SP must diffuse through even larger pieces of undissolved film leading to more pronounced losses during its extraction.

An important aspect of pharmaceutical dosage forms is the solid state of the API since it influences the solubility and thus bioavailability (Lust et al., 2013; Martínez et al., 2014). 
Using the design of the samples characterized by optical and electronic microscopies, the amounts of printed SP were below the detection limit of SWAXS and DSC which also happened in other studies regarding printed APIs (Buanz et al., 2011). Printing larger amounts of SP enabled its detection with DSC. However, the absence of crystallization peak in the SWAX diffractograms must be carefully interpreted since the printed amounts of SP may have remained too close to the detection limit. Printed SP was detectable with polarized light microscopy and no indication of crystalline areas within the printed API on any of the eight substrates was found with PLM. This might be due to the small volume of the printed aqueous ink. The deposited drops had a volume of 7.2-8.6 $\mathrm{nl}$ and therefore, the solvent evaporated too rapidly to leave the drug molecules enough time to organize, resulting in the formation of amorphous solid dots of SP. When printed on glass slides, SP only crystallized if higher volumes of ink were printed on the same area and drops coalesced to a combined volume of about $4.5 \mu \mathrm{l}$. Buanz et al. (2015) compared oral films of clonidine hydrochloride with the drug either incorporated into the films by solvent casting or printed on top of the films (Buanz et al., 2015). Crystallization of the drug was only found in the cast films and not when smaller amounts of ink were printed allowing the solvent to evaporate quickly. However, humidity may have an influence on long term stability.

The different substrates showed different release profile of SP from the printed dosage forms, although the API was primarily located on the surface of the films. As expected, the fast dissolving films, Tesa and List, caused faster drug release and gelling polymers, such as GE and HPMC, which caused slower release. Tesa and List disintegrated rapidly causing an increase in specific surface area and therefore, SP was dissolved quickly. GE and HPMC formed clumps at the bottom of the dissolution vessel which may have entrapped SP causing 
slower drug release. Interestingly, the addition of $2 \% \mathrm{TiO}_{2}$ to GE or HPMC accelerated the release and raised the maximum amount of released drug by 5\%. pMCC did not disintegrate at all and yMCC disintegrated into small particles forming an opaque suspension during dissolution. However, the drug release from the MCC films was the fastest of all eight films. This may happen due to two reasons: firstly, SP is visible on the surface of MCC films when freshly printed and secondly, MCC films are porous (Planchette et al., 2016). Molecules situated at the surface of the film and in pores are in contact with the liquid bulk in which they are released without diffusing through the solid matrix. This could be responsible for faster release.

Model APIs with different crystallization behavior need to be investigated regarding their behavior when printed onto different substrates. The impact of $\mathrm{TiO}_{2}$ on wettability and dissolution needs to be elucidated further.

\section{Conclusion}

Ink jet printing of aqueous SP inks provides good stability and content uniformity by generating stable drop volume over contactless jetting. The morphology and size of the printed API drops depend on the surface of the substrates and it is crucial to understand the interactions between the API and the substrates to ensure good dosage form quality. The investigated substrates did not lead to the formation of crystalline domains within dried SP drops in contrast to inert substrate such as glass. Thus, the investigated substrates can be considered as efficient excipients to inhibit the undesired crystallization of SP under the conditions we have been using them. Furthermore, no chemical interactions were observed 
between the API and the films. The study showed that the drug release of printed APIs can

be influenced by the nature of the substrate and that printed APIs can be monitored for quality analysis purposes with OCT and standard methods. All substrates investigated in this study can be employed to be a printing substrate for SP.

\section{Acknowledgements}

This work has been funded within the Austrian COMET Program under the auspices of the Austrian Federal Ministry of Transport, Innovation and Technology (BMVIT), the Austrian Federal Ministry of Economy, Family and Youth (BMWFJ) and by the State of Styria (Styrian Funding Agency SFG). COMET is managed by the Austrian Research Promotion Agency FFG. We thank our colleagues at the Research Center Pharmaceutical Engineering for technical support.

\section{References}

Adler, C., Schönenberger, M., Teleki, A., Leuenberger, B., Kuentz, M., 2015. Journal of Pharmaceutical and Biomedical Analysis Flow-through cross-polarized imaging as a new tool to overcome the analytical sensitivity challenges of a low-dose crystalline compound in a lipid matrix. J. Pharm. Biomed. Anal. 115, 20-30. https://doi.org/10.1016/j.jpba.2015.06.033

Alomari, M., Mohamed, F.H., Basit, A.W., Gaisford, S., 2015. Personalised dosing: Printing a dose of one's own medicine. Int. J. Pharm. 494, 568-577. https://doi.org/10.1016/j.ijpharm.2014.12.006

Berezin, K. V, Nechaev, V. V, Zotov, S.N., 2004. Hydrogen Bond Effects on the Fundamental Vibration Frequencies of Pyridine 45, 412-418.

Berthuy, O.I., Blum, L.J., Marquette, C.A., 2016. Cancer-cells on chip for label-free detection of secreted molecules. Biosensors 6. https://doi.org/10.3390/bios6010002

Boehm, R.D., Miller, P.R., Daniels, J., Stafslien, S., Narayan, R.J., 2014. Inkjet printing for pharmaceutical applications. Mater. Today 17. https://doi.org/10.1016/j.mattod.2014.04.027

Buanz, A.B.M., Belaunde, C.C., Soutari, N., Tuleu, C., Gul, M.O., Gaisford, S., 2015. Ink-jet printing versus solvent casting to prepare oral films: Effect on mechanical properties and physical stability. Int. J. Pharm. 494, 611-618. https://doi.org/10.1016/j.ijpharm.2014.12.032

Buanz, A.B.M., Saunders, M.H., Basit, A.W., Gaisford, S., 2011. Preparation of personalized-dose salbutamol sulphate oral films with thermal ink-jet printing. Pharm. Res. 28, 2386-2392. https://doi.org/10.1007/s11095-011-0450-5

Daly, R., Harrington, T.S., Martin, G.D., Hutchings, I.M., 2015. Inkjet printing for pharmaceutics - A review of research and manufacturing. Int. J. Pharm. 494.

https://doi.org/10.1016/j.ijpharm.2015.03.017 
Edueng, K., Mahlin, D., Larsson, P., Bergström, C.A.S., 2017. Mechanism-based selection of stabilization strategy for amorphous formulations: Insights into crystallization pathways. J. Control. Release. https://doi.org/10.1016/j.jconrel.2017.04.015

EMA, 2013. European Pharmacopoeia - 8th Edition.

Fraser, S.D., Khinast, J.G., Bauer, W., Brenn, G., Zimmer, A., 2013. Das Drucken patientenspezifischer pharmazeutischer Formulierungen. Techno Pharm 3, 246-252.

Garsuch, V., Breitkreutz, J., 2009. Novel analytical methods for the characterization of oral wafers. Eur. J. Pharm. Biopharm. 73, 195-201. https://doi.org/10.1016/j.ejpb.2009.05.010

Genina, N., Fors, D., Palo, M., Peltonen, J., Sandler, N., 2013. Behavior of printable formulations of loperamide and caffeine on different substrates - Effect of print density in inkjet printing. Int. J. Pharm. 453, 488-497. https://doi.org/10.1016/j.ijpharm.2013.06.003

George Socrates, 2004. Infrared and Raman Characteristic Group Frequencies, 3rd ed. Wiley.

Hager, H., Blaschek, W., Ebel, S., Hackenthal, E., 2003. HagerROM, 3rd ed. Springer Science \& Business Media.

H. Hsu, S. Toth, G. Simpson, L. Taylor, M.H., 2013. Effect of Substrates on Naproxen-Po lyvinylpyrro lidone Solid Dispersions Fo rmed via t he Drop Printing Technique. J. Pharm. Sci. 102, 638-648.

Janßen, E.M., Schliephacke, R., Breitenbach, A., Breitkreutz, J., 2013. Drug-printing by flexographic printing technology - A new manufacturing process for orodispersible films. Int. J. Pharm. 441, 818-825. https://doi.org/10.1016/j.ijpharm.2012.12.023

Kastner, J., Faury, T., Außerhuber, H.M., Obermüller, T., Leichtfried, H., Haslinger, M.J., Liftinger, E., Innerlohinger, J., Gnatiuk, I., Holzinger, D., Lederer, T., 2017. Silver-based reactive ink for inkjetprinting of conductive lines on textiles. Microelectron. Eng. 176, 84-88. https://doi.org/10.1016/j.mee.2017.02.004

Li, S., Zhang, Q., Lu, Y., Ji, D., Zhang, D., Wu, J., Chen, X., Liu, Q., 2016. One step electrochemical deposition and reduction of graphene oxide on screen printed electrodes for impedance detection of glucose. Sensors Actuators B Chem. 244, 290-298. https://doi.org/10.1016/j.snb.2016.12.142

Lin, H., Dong, Y., Markl, D., Zhang, Z., Shen, Y., Zeitler, J.A., 2017. Pharmaceutical Film Coating Catalog for Spectral Domain Optical Coherence Tomography. J. Pharm. Sci. 106, 3171-3176. https://doi.org/10.1016/j.xphs.2017.05.032

Lust, A., Laidmäe, I., Palo, M., Meos, A., Aaltonen, J., Veski, P., Heinämäki, J., Kogermann, K., 2013. Solid-state dependent dissolution and oral bioavailability of piroxicam in rats. Eur. J. Pharm. Sci. 48, 47-54. https://doi.org/10.1016/j.ejps.2012.10.005

Markl, D., Hannesschläger, G., Sacher, S., Leitner, M., Khinast, J.G., 2014. European Journal of Pharmaceutical Sciences Optical coherence tomography as a novel tool for in-line monitoring of a pharmaceutical film-coating process. Eur. J. Pharm. Sci. 55, 58-67. https://doi.org/10.1016/j.ejps.2014.01.011

Martínez, L.M., Videa, M., López-Silva, G.A., De Los Reyes, C.A., Cruz-Angeles, J., González, N., 2014. Stabilization of amorphous paracetamol based systems using traditional and novel strategies. Int. J. Pharm. 477, 294-305. https://doi.org/10.1016/j.ijpharm.2014.10.021

Pardeike, J., Strohmeier, D.M., Schrödl, N., Voura, C., Gruber, M., Khinast, J.G., Zimmer, A., 2011. Nanosuspensions as advanced printing ink for accurate dosing of poorly soluble drugs in personalized medicines. Int. J. Pharm. 420, 93-100. https://doi.org/10.1016/j.ijpharm.2011.08.033

Planchette, C., Pichler, H., Wimmer-Teubenbacher, M., Gruber, M., Gruber-Woelfler, H., Mohr, S., Tetyczka, C., Hsiao, W.K., Paudel, A., Roblegg, E., Khinast, J., 2016. Printing medicines as orodispersible dosage forms: Effect of substrate on the printed micro-structure. Int. J. Pharm. 509, 518-527.

Preis, M., Breitkreutz, J., Sandler, N., 2015. Perspective: Concepts of printing technologies for oral film formulations. Int. J. Pharm. 494, 578-584. https://doi.org/10.1016/j.ijpharm.2015.02.032

Quéré, D., 2008. Wetting and Roughness. Annu. Rev. Mater. Res. 38, 71-99. https://doi.org/10.1146/annurev.matsci.38.060407.132434 
Romani, D., Tonello, I.S., Brandán, S.A., 2016. Influence of atomic bonds on the properties of the laxative drug sodium picosulphate. Heliyon 2, e00190. https://doi.org/10.1016/j.heliyon.2016.e00190

Sandler, N., Preis, M., 2016. Printed Drug-Delivery Systems for Improved Patient Treatment. Trends Pharmacol. Sci. 37, 1070-1080. https://doi.org/10.1016/j.tips.2016.10.002

Vakili, H., Nyman, J.O., Genina, N., Preis, M., Sandler, N., 2016. Application of a colorimetric technique in quality control for printed pediatric orodispersible drug delivery systems containing propranolol hydrochloride. Int. J. Pharm. 511, 606-618. https://doi.org/10.1016/j.ijpharm.2016.07.032

Wittbrodt, B., Pearce, J.M., 2017. 3-D printing solar photovoltaic racking in developing world. Energy Sustain. Dev. 36, 1-5. https://doi.org/10.1016/j.esd.2016.08.001

Zhang, S.-W., Yu, L., Huang, J., Hussain, M. a, Derdour, L., Qian, F., de Villiers, M.M., 2014. A method to evaluate the effect of contact with excipients on the surface crystallization of amorphous drugs. AAPS PharmSciTech 15, 1516-26. https://doi.org/10.1208/s12249-014-0178-8 